\title{
Veränderte Prozesse der Entscheidungsfindung in der Raumentwicklung
}

Joris Van Wezemael, Freiburg, Matthias Loepfe, Zürich

\begin{abstract}
«Wenn sich die Beteiligten darüber einig sind, was sie wollen, und wie dies zu erreichen ist, dann überwiegt die Sicherheit, und Planung entspricht der Anwendung rationalen Wissens. Wenn sie sich darüber einig sind, was sie wollen, aber nicht wissen, wie dies zu erreichen ist, dann wird Planung zu einem Lernprozess. Sind sich die Beteiligten darüber uneins, was sie erreichen wollen, wissen aber, wie die jeweiligen Alternativen umsetzbar sind, dann ist Planung ein Verhandlungsprozess. Sind sie sich sowohl über Mittel als auch Zweck uneins, so wird Planung zu einer Suche nach Ordnung im Chaos. Jede prototypische Situation verweist auf eine bestimmte Bandbreite von Planungsstilen. Planer sollten daher ihre Vorgehensweisen den jeweiligen Situationen anpassen. Indem sie jeweils den Kontingenzen entsprechend handeln, können sie auf Basis von Vernunft mit Unsicherheiten umgehen» (Christensen 1985: 63; Übers. d. Verf.).
\end{abstract}

\section{Planung und Unsicherheit}

In ihrem viel beachteten Artikel von 1985 charakterisiert Karen S. Christensen (siehe einleitendes Zitat) Probleme der Planung nach deren Verhältnis hinsichtlich der Unsicherheit ihrer Ziele und den Mitteln, um diese zu erreichen. Sind die Ziele und die Mittel zur Erreichung - auch «Technologie» genannt - bekannt, so kann eine Abwicklung der Planung erfolgen. Herrscht indes kein Zielkonsens, so müssen Ziele verhandelt werden. Sind die Mittel zur Zielerreichung nicht bekannt, kommen Experimente zum Zug. Die Komplexität nimmt weiter zu, wenn sowohl Uneinigkeit über die Ziele herrscht als auch mögliche Lösungswege unsicher erscheinen. Gemäss De Roo \& PorTer (2006) ergibt sich aus Christensen's Ansatz ein Kontinuum von Planungsproblemen, die sich in ihrem Grad an Unsicherheit unterscheiden. Der Grad an Unsicherheit wiederum widerspiegelt deren Grad an Komplexität. Hierauf Bezug nehmend argumentiert VAN Wezemael (2009b), dass Planungsprobleme über wiederkehrende Versuche der Problemlösung und über eine Abfolge von Scheitern und erneutem Versuch die Planungsprozesse an einen Grad von Komplexität heranführen, der im Sinne einer Systemsteuerung (Ashby 1956) problemangemessen ist. Diese These soll an empirischem Material ausgeführt und getestet werden.
Diese Perspektive rückt den experimentellen Charakter von Planung im Sinne von ex ante gerichteten Handlungsrahmen ins Zentrum, sodass jede «Lösung» als nur temporäre oder provisorische «Fixierung eines Flux» verstanden wird. De Roo \& PORTER (2006) spannen ein Kontinuum auf zwischen technischer Rationalität für relativ überschaubare Probleme und kommunikativen Ansätzen für Situationen, die von grosser Unsicherheit bestimmt sind. Es wird argumentiert, dass Entscheidungsmodi sich in ihrem Freiheitsgrad über einen experimentellen trial and error-Prozess dem Komplexitätsgrad des Problems annähern. Ein Freiheitsgrad bezeichnet generell einen Parameter eines Systems und ist eine Systemeigenschaft. In einem physikalischen System versteht man darunter eine Koordinate, mit der das System beschrieben werden kann; bei Gelenken beschreibt der Freiheitsgrad die Anzahl und Art der möglichen Bewegungen, die das Gelenk ausführen kann. Freiheitsgrade bezeichnen also einen Möglichkeitsraum. Der Komplexitätsgrad ist hierbei als eine emergente Eigenschaft zu verstehen, die durch die Planungssituation selbst generiert wird. Für die Planungstheorie liegt die Herausforderung also darin, die Offenheit und Unsicherheit in Planungsverfahren konzeptionell fassen zu können. Hierzu soll der vorliegende Artikel einen Beitrag leisten.

Wesentliche Herausforderungen aktueller Planung wie Entwicklungen im Bestand, die Bildung von regionalen Netzwerken oder die Aktivierung von Potentialen (MAYER 2004: 134) zeichnen sich durch einen hohen Grad von Ressourceninterdependenz der Beteiligten aus. In veränderten relationalen Settings sind Ressourcen über verschiedene Akteure verteilt: Verfahrenskompetenz, Weisungsbefugnis, Know-how, Investitionskapital, Positionen in machtvollen Netzwerken. Akteure lassen sich deshalb auf einen zunächst offenen Aushandlungsprozess ein, weil jeder beteiligte Akteur nicht alleine an sein Ziel gelangen kann, und weil die Unsicherheiten bezüglich der Aktionen anderer Akteure zu gross sind (JESSOP 1999). Rollen werden neu definiert, Aufgaben müssen erst noch erkundet werden, Ziele sind anfangs unklar (SCHOLl 1995). Die Folge hiervon ist Unsicherheit hinsichtlich der Ziele, Verfahren und Akteure. Der Umgang mit Unsicherheit führt dazu, dass die Zielaushandlung und der experimentelle Prozess von trial and error die Planung zusehends vom Phasenmodell (siehe auch Beitrag IBERT in diesem Heft) entfernt; sie findet zunehmend in konkreten Projek- 
ten statt. Hiermit geht eine Hinwendung zu einem prozess-, umsetzungs- und aufgabenorientierten Planungsverständnis einher, welches in der Planungspraxis nach dem Scheitern weisungsbasierter Planungsmodi oft zur Schaffung von ad hoc-Organisationen führt. Vertreter der deutschsprachigen Debatte der «Projektorientierung» (vgl. z.B. IBERT 2003; Keller et al. 1996; MAYER 2004; RoMmelsPaCher 1999; Siebel et al. 1999) argumentieren, dass sich die öffentliche Hand vermehrt über rahmensetzende Pläne hinaus umsetzungsorientiert in Projekten engagieren soll. Die «Aktionsplanung» (ScHOLL 1995) trägt der Komplexität in der Raumentwicklung insofern Rechnung, als betont wird, dass Organisationsformen sich nach den Aufgaben richten sollen und nicht umgekehrt. Die angelsächsische «Governance»-Forschung (vgl. Healey 2005; Jessop 1998, 1999) wiederum betont das Auftreten neuer «Governance»-Formen, welche die beiden idealtypischen Koordinationsmechanismen der staatlich-hierarchischen Verordnung und der marktwirtschaftlichen Logik der Effizienz ergänzen und überlagern.

Der vorliegende Artikel setzt sich, ausgehend von einer sowohl in der Planungspraxis als auch im planungstheoretischen Diskurs zunehmenden «Projektorientierung», zum Ziel, (1) planerische Lösungsversuche als komplexe Prozesse kollektiver Entscheidungsfindung zu konzipieren, (2) sie empirisch in ihrer Entfaltung nachzuzeichnen, und (3) deren bestimmende Momente zu analysieren. Hierzu werden drei aktuelle, kontextuell deutlich unterschiedliche Planungsprojekte aus der Schweiz im Sinne von Entscheidungsprozessen nachgezeichnet. Besondere Beachtung findet hierbei die Frage, welche «Möglichkeitsräume» im Sinne potentieller Prozessverläufe aus der Konstellation von Prozesskomponenten generiert werden, und wie diese den weiteren Verlauf der Entscheidungsfindung mitbestimmen. Zur Konzeption der Planungsprozesse wird mit der Assemblage-Theorie (DeLanda 2006) ein Ansatz zur Diskussion gestellt, der in der deutschsprachigen Debatte bislang noch kaum Aufmerksamkeit erfahren hat. Der Beitrag der Theorie von Assemblages und den Prozessen, die sie generieren und transformieren, liegt darin, dass sie intermediäre Einheiten jenseits von Mikro- und Makro-Konzeptionen wie Organisationen, Netzwerken und Städten ontologisch reflektiert, dass sie die Offenheit und Unsicherheit in Planungsprozessen als zentrale Eigenschaft von Assemblages anspricht, ferner, dass sie Emergenz erfasst und so eine dynamische Perspektive sowie eine Dimension des Werdens in die Analyse einbringt, und dass sie globalisierte Zusammenhänge in lokalen Entscheidungsprozessen adäquat thematisiert.

Schlussfolgernd stellt sich der vorliegende Beitrag die derzeit politisch brisante Frage - die Revision des
Raumentwicklungsgesetzes befindet sich in Vernehmlassung - nach dem Verhältnis veränderter Entscheidungsprozesse zu den bestehenden rechtlichen Rahmenbedingungen.

\section{Konzeptioneller Zugang}

Um gewandelte Modi der Entscheidungsfindung, die Etablierung von ad hoc-Organisationen und die resultierenden Dynamiken in der Raumentwicklung analysieren zu können, wurden aktuelle Fallbeispiele einer Prozessanalyse unterzogen. Durch Konzipierung von Planungen als nicht-lineare Prozesse werden Phasen sichtbar, in denen Entscheide mit vergleichsweise hohen Freiheitsgraden getroffen werden können und solche, in denen nur wenige Optionen zur Auswahl stehen.

Es wird argumentiert, dass Planungsprozesse in verschiedenen Projekten deshalb individuell verlaufen, weil sie aus der jeweiligen Situation im Sinne eines Netzwerkes von Orten, Akteuren, juridischen Bestimmungen, Repräsentationen (z.B. Pläne) jeweils spezifisch - als Individuen (siehe unten) - generiert werden. Die Argumentation für eine Aufgabenorientierung in der Planung nach Scholl (1995) ist in diesem Sinne zu lesen. In der planungstheoretischen Debatte wird zudem argumentiert, dass aktuelle Prozesse der Raumentwicklung durch Nicht-Linearität, Unsicherheiten und plötzliche Umbrüche geprägt sind (Albrechts \& Mandelbaum 2006; Amin 2004; Bertolini 2007; Christensen 1985; De Roo \& Porter 2006; Hillier 2006; Hillier \& Van Wezemael 2008c; Van WezeMAEL 2008). Methodisch und konzeptionell stellt sich daher die Frage, wie man die veränderten Prozesse der Raumentwicklung fassen und erklären kann. LAw \& URRY (2004: 390) argumentieren, dass

«[...] Wandel in der sozialen und physischen Welt vom Wandel in den Methoden der Sozialwissenschaften begleitet wird - und das ist gut so» (Übers. d. Verf.).

Komplexitätstheoretische Analysen beruhen auf den Dynamiken von Ungleichgewichtszuständen und der Betonung von multiplen Zukünften, historischer Pfadabhängigkeit und intrinsischer Unsicherheit. Planungssettings werden durch Interaktion, Nicht-Linearität, Instabilität, Unvorhersehbarkeit und Selbstorganisation beschrieben; eine gegebene Ursache eröffnet Potentiale, die durch generative Prozesse in Möglichkeiten überführt werden. Das Leitmotiv der Complexity Sciences beschreibt, dass jegliche beobachtbare Ordnung - also auch jede räumliche Konfiguration und jedes soziale Ensemble - als Produkt der Interaktionen von Elementen hervortritt. Ein Individuum wird immer als Prozess verstanden, wobei Individuen alle semi-stabilen Einheiten umfassen, welche die 
Kapazität haben, um etwas zu bewirken (siehe unten). Komplexitätsdenken fordert dazu auf, so vielschichtige und dynamische Phänomene wie Siedungsräume als verflochtene Systeme mit menschlichen und materiellen Komponenten anzuerkennen und nicht auf eine anthropozentrische Sicht zu reduzieren (AlEXANDER 2002; DurLauf 2005; Summers-EFfler 2007).

Die Varietät des Untersuchungsgegenstandes verlangt daher nach einem Ansatz, der unvoreingenommen - also ohne die Projektion vordefinierter Verantwortlichkeiten und Abläufe - an die Empirie herantritt. Die in rezenten stadtgeographischen und planungswissenschaftlichen Arbeiten zur Diskussion gestellte Assemblage-Theorie von Manuel DeLanda (2006) wird als adäquater Zugang erachtet (Hillier \& VAN Wezemael 2008a, 2008b; Van Wezemael 2009a, 2009b). Die AssemblageTheorie ist ein konstruktiv-realistischer Ansatz, der soziale Ensembles auf Basis der Prozesse, die sie generieren, anspricht. Sie konzeptionalisiert Prozesse der Kreation und Transformation von sozialen Ensembles, die als Assemblages bezeichnet werden, und unterbreitet eine Herangehensweise zur Analyse generativer Prozesse. Der Ansatz zeichnet sich dadurch aus, dass potentiellen Prozessen des Werdens von Assemblages explizit und auf einer soliden wissenschaftstheoretischen Basis Beachtung geschenkt wird (DeLANDA 2006). Hier geht der Ansatz auf Deleuze's (1994) Mahnung ein, wonach nichts verstanden werden kann, indem man es auf seine aktuell gegebenen Eigenschaften reduziert. Vielmehr ist zu der Ebene des Aktualisierten immer eine virtuelle Ebene sowie ein generatives Feld zu denken, welche die Tendenzen zum Wandel und Werden einer Sache und deren aktuell nicht manifestierten Qualitäten enthält. Diese werden im Folgenden vereinfachend als «Möglichkeitsräume» bezeichnet. Konsequenterweise stellt sich in der vorgeschlagenen Perspektive nicht die (essenzialistische) Frage, was ein Ensemble ist, sondern diejenige, was es «be-wirken» kann.

In konkreten Studien geht es darum, Assemblages als vorerst abstrakte Konzeption von kollektiven Akteuren zu konzipieren, was empirisches Arbeiten dazu zwingt, jene Relationen zu benennen, die emergente Eigenschaften der Ensembles hervorbringen. Das heisst, dass Begriffe wie «Projektorientierung» nur postuliert werden sollten, wenn die Prozesse angegeben werden können, welche entsprechende Assemblages als historische Individuen generieren. «Historisch» betont hier, dass die Prozesse ihrer Genese, ihrer Reproduktion und ihrer Transformation benannt werden müssen. So lauten die relevanten Fragen: Wie wird ein Ensemble generiert? Wie wird dessen Identität stabilisiert oder transformiert?

Stabilisieren kann in Anlehnung an Gilles Deleuze und Felix Guattari (1987) als Territorialisierung bezeichnet werden. Diese räumliche Metapher bezieht sich auf Prozesse, welche die Grenzen von Entitäten schärfen (z.B. «wir» versus «die Anderen», Abgrenzung von Stadtquartieren) respektive deren innere Homogenität erhöhen. De-Territorialisierung meint entsprechend das Aufbrechen fixierter Beziehungen, eine Zunahme der inneren Heterogenität von Assemblages oder eine Schwächung der Identität von Gruppen oder Orten.

Planung befasst sich in erster Linie mit Entitäten, die in die Bruchstellen von Mikro- und Makroansätzen fallen. Hier leistet die Assemblage-Theorie einen bedeutenden Beitrag, indem sie individuelle Personen, Populationen von Personen, Freundschaftsnetzwerke, Organisationen, inter-organisationale Netzwerke, Quartiere, Städte als historisch produzierte Individuen mit emergenten Eigenschaften konzipiert (sie sind emergente soziale Formationen, die sich auf Basis der Interaktionen ihrer Komponenten ergeben). Sie verfügen somit alle über denselben ontologischen Status. Ihre Komponenten sind in jedem Falle ebenso als Assemblages zu verstehen.

Die relevanteste Veränderung der Planung betrifft auf Basis der bisherigen Argumentation die Zunahme von Akteuren und das Aufsprengen raumzeitlicher Grenzen. Die Frage nach den relevanten Akteuren beantwortet die Assemblage-Theorie analog zu Latour's Akteurverständnis (2007: 71):

«Man muss nur folgende Frage stellen: bewirkt das fragliche Objekt etwas im Handlungsverlauf eines anderen Akteurs oder nicht?» (Übers. d. Verf.)

Das heisst für die Analyse von Projektplanungen, dass alles, also auch Infrastrukturen und entfernte Orte, darauf geprüft werden muss, was sie bei anderen Akteuren «be-wirken». Kann man sie nicht ohne Konsequenzen aus einem Tätigkeitsprozess entfernen, so gehören sie zum kollektiven Akteur im Sinne eines Assemblage und dessen Kapazitäten, etwas zu «bewirken».

Im vorliegenden Beitrag folgt die Analyse der Fallbeispiele dem Erkenntnisinteresse daran, was Investoren, Pläne, Infrastrukturen, Gesetze im Sinne von interagierenden Komponenten eines Assemblage in den untersuchten Projekten bewirken, und wie die durch die spezifischen Konstellationen generierten Möglichkeitsräume den weiteren Planungsprozess formen.

Im Sinne einer Heuristik wurde Kipp-Punkten im Prozessverlauf eine besondere Aufmerksamkeit geschenkt. Es handelt sich dabei um Momente, welche den aktuellen Planungsprozess und seine virtuellen Potentiale auf entscheidende Weise beeinflusst haben und somit als singulär im Prozess gelten dürfen. An 
Kipp-Punkten werden Beziehungen zwischen Komponenten des Assemblage so modifiziert, dass sich im Sinne eines Schwellenwerts eines Systems qualitativ veränderte Dynamiken manifestieren können. Das Projekt als Assemblage ist an dieser Stelle äusserst fragil: Kleine Änderungen (der Systemparameter) können grosse Folgen für den Planungsprozess und seine virtuellen Potentiale haben; bisherige Lösungswege werden destabilisiert und eventuell neue eingeschlagen. Die Assemblage-Theorie soll im Zuge der empirischen Untersuchung weiter ausgeführt und deren Vorzüge aufgezeigt werden.

Kern der Datenerhebung war eine Abfolge von Workshops, in denen die Projekte von in Voruntersuchungen eruierten Schlüsselpersonen vorgestellt, die Fragestellung in einem weiteren Kreis von Experten diskutiert und schliesslich die Resultate der jeweiligen Datenauswertung validiert wurden. Die empirische Basis bildeten audiovisuelle Aufzeichnungen der Workshops und Originaldokumente der untersuchten Projekte.

Durch die Arbeit mit Originalunterlagen konnte beispielsweise die Skalierung von Projekten als Errungenschaft der im Projekt interagierenden Komponenten - und nicht als vorbestimmter Perimeter - entschlüsselt werden. In einer von der AssemblageTheorie angeleiteten qualitativen Inhaltsanalyse wurden Kipp-Punkte herausgearbeitet sowie dadurch initiierte Prozesse der De-/Re-Territorialisierung und Veränderungen von Möglichkeitsräumen nachvollzogen. Ein besonderes Augenmerk wurde darauf gelegt, wie planerische Lösungsversuche mit Unsicherheiten umgingen, und welche prozessualen und organisatorischen Folgen dies zeitigt. Die Auswertung wurde von der Software Atlas.ti unterstützt. Sie erleichterte die Zuordnung von Aussagen und Repräsentationen zu den entsprechenden Kipp-Punkten.

\section{Planerische Lösungsversuche und Kipp-Punkte}

In den folgenden Abschnitten wird ein Einblick in die Ergebnisse der retrospektiven Analyse der Fallbeispiele Bern Brünnen, Cornaredo Lugano und Parc Ela gegeben. Planerische Lösungsversuche als komplexe Prozesse kollektiver Entscheidungsfindung werden empirisch anhand von Kipp-Punkten, der Veränderung von Möglichkeitsräumen sowie Prozessen der De-/ReTerritorialisierung nachgezeichnet.

\subsection{Bern Brünnen}

Die Planung auf dem Gebiet Bern Brünnen hat eine fast 40-jährige Geschichte. Planungsvorhaben verschiedener Ausrichtung waren schon mehrmals gescheitert - der trial and error-Prozess hat eine lange Geschichte. Das so genannte «Approximative Generalkonzept
Brünnen» von 1972 - es sah eine Stadterweiterung mit Wohnraum für 20'000 Menschen vor (vgl. Abb. 1) - landete aufgrund der Rezessionsphase während der Ölkrise sowie der Trendwende von der Landflucht zur Suburbanisierung im Archiv. Eine redimensionierte Fassung wurde 1978 wieder aufgenommen, scheiterte aber in einer Abstimmung vor dem Volk. Erfolgsversprechender erschien die Idee einer Teilüberbauung von Brünnen Nord und der gleichzeitigen Auszonung von Brünnen Süd, welche vom Stimmvolk 1991 gut geheissen wurde. Der Zusammenbruch des Immobilienmarktes führte aber dazu, dass keine Investoren gefunden wurden. Die Möglichkeiten der Entwicklung wurden zudem limitiert durch hohe Kosten für Lärmschutzmassnahmen (Autobahnüberdeckung), welche durch die Lage an der Autobahn und die Lärmschutzverordnung (LSV) zwingend notwendig waren. Durch die Zahlungsunfähigkeit zweier Eigentümer, welche die teure Infrastruktur tragen sollten, wurde eine Realisierung im Sinne der geplanten Überbauungsordnung immer unwahrscheinlicher. Die Gemeinde Bern und der Bund, welcher im Übrigen über das Wohneigentumsförderungsgesetz (WEG) an einer der betroffenen Immobiliengesellschaften beteiligt war, gerieten unter Handlungsdruck. Die verfahrene Situation löste eine Reihe von Aktivitäten zwischen der öffentlichen Hand, Immobilienfirmen, Ökonomen und Architekten im lokalen Umfeld der Stadt aus. An dieser Stelle ist ein wichtiger Kipp-Punkt auszumachen, an welchem der Prozess durch private Initiative in eine neue Richtung gelenkt wurde. So brachte sich ein mit dem vorgängigen Projekt vertrauter Ingenieur mit einem Lösungsvorschlag für die Finanzierung der Autobahnüberdeckung in die Diskussion ein, welcher einen Möglichkeitsraum aufspannte, in welchem die Elemente des Brünnener Planungsensembles veränderte Rollen spielten. Die Idee beinhaltete im Wesentlichen, neben den reduzierten Wohnflächen eine grössere kommerzielle Nutzfläche zu realisieren. Mit der Schaffung von attraktiveren Bedingungen für Investoren wurde die Siedlungsentwicklung im Gebiet Bern Brünnen wieder möglich. Die neuen Entwicklungsmöglichkeiten liessen verschiedene Beteiligte sowie auch bisher Unbeteiligte in veränderten Relationen miteinander in Beziehung treten. So wurde eine potentielle Verbindung zu einem starken Investor aktualisiert, indem ein lokaler Immobilienentwickler ein Interesse an der Entwicklung eines überregionalen Freizeit- und Einkaufszentrums weckte. Die öffentliche Hand begann, auf Basis der veränderten Entwicklungsmöglichkeiten mit dem privaten Investor zu kommunizieren, was einen Austauschprozess auslöste und den Prozess der Entscheidungsfindung vorantrieb.

An dieser Stelle kann ein grundlegendes Konzept der Assemblage-Theorie ausgeführt werden: Sie ersetzt die vorherrschende sozialwissenschaftliche Konzep- 


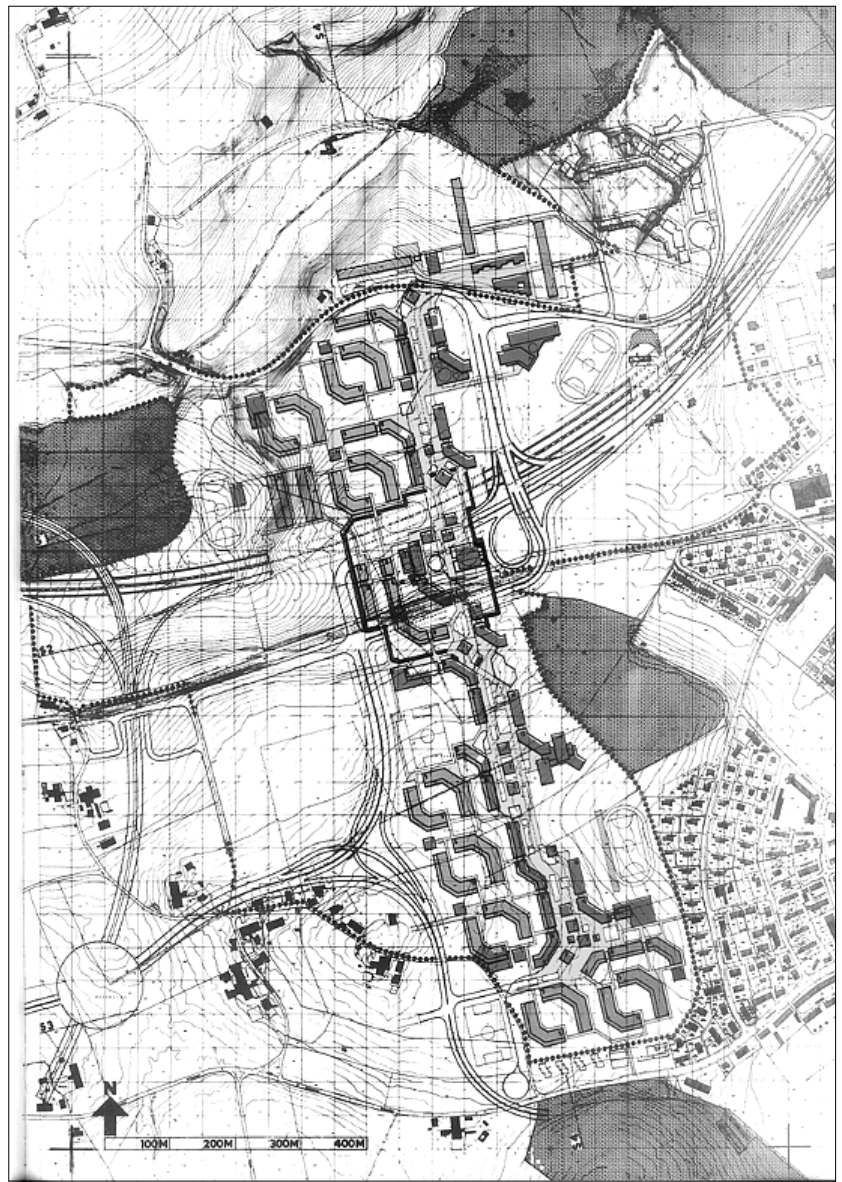

Abb. 1: Approximatives Generalkonzept Bern Brünnen, Schweiz

Basic general concept Bern Brünnen, Switzerland

Approximation du concept général de Berne Brünnen, Suisse

Quelle: Archiv Stadtplanungsamt Bern, 1972 (mit freundlicher Genehmigung)

tion von so genannt interioren durch exteriore Beziehungen. Während interiore Beziehungen implizieren, dass die Komponenten durch die Rolle, die sie hinsichtlich des Ganzen spielen, konstituiert sind, erhalten Komponenten in exterioren Relationen eine grössere Autonomie. Denn ihre Beziehungen können sich ändern, ohne dass die Komponenten ihre konstitutive Basis verändern (DeLanda 2006: 9-11). Wird ein Element in einem durch interiore Relationen verstandenen Ganzen entfernt, so verliert es gleichsam seine Identität, weil diese wesentlich darin bestand, Teil des Ganzen zu sein (DeLanda 2006: 9). Ein Element eines Ganzen hingegen, welches durch exteriore Relationen konstituiert ist, kann aus einem Ganzen herausgelöst werden und mit Komponenten anderer Entitäten in Beziehung treten (DeLanda 2006: 10). Das heisst, dass dieselben Elemente gleichzeitig Teil verschiedener und oft sehr unterschiedlicher Assemblages sind. Die Betonplatte der Autobahnabdeckung wurde gleichzeitig zu einem kalkulativen Element im «Rendite-Assemblage» aus Arealentwicklern und Investoren und zu einem Element im politisch-planerischen Assemblage rund um den Lärmschutz. Sie generierte durch ihre Relation mit den unterschiedlichen Assemblages ein Set von Optionen, die zuvor nicht bestanden. Das materielle Element «Betonplatte» wurde gemäss Definition zu einem vollwertigen Akteur, weil es Andere dazu veranlasste, etwas zu tun. 
Erst die aufgrund der neuen Relationen veränderten Potentiale erlaubten den Miteinbezug und Einstieg des privaten, finanzstarken Investors. Die Potentiale der Entwicklung wandelten sich derart, dass eine Arealentwicklung wieder möglich, jedoch eine Überbauung mit Wohnbauten im bisherigen Ausmass gleichzeitig eingeschränkt wurde. De-Territorialisierung und ReTerritorialisierung sind also nicht Gegensätze - vielmehr bedingen sie einander. Unsicherheiten bezüglich der Realisierung wurden durch die Zusage eines finanzstarken Investors reduziert. Dadurch wurde das generative Feld neu strukturiert, und die Prozesse der Aktualisierung von Lösungen wandelten sich. Zum Einen wurde der bisherige ex ante gerichtete Handlungsrahmen (mit einer hohen Anzahl von Wohnungen) erschüttert oder eben: de-territorialisiert. Zum Anderen gewinnen spezifische Relationen zwischen den Entwicklern, der öffentlichen Hand und dem privaten Investor an Relevanz. Die Verbindung von materiellen, ökonomischen und politisch-planerischen Elementen generiert eine Dynamik, welche sowohl die Abläufe als auch die (diskursive) Rahmung (AmIN 2004) des Projektes re-territorialisiert.

Die bisherige Konzeption des Ortes als Wohngebiet wurde durch die Verbindung mit diesen neuen Elementen schon in der Anfangsphase des Projektes de-/re-territorialisiert und virtuell neu generiert: Die Konzeption wandelte sich von einem reinen Wohnquartier zu einem Entwicklungsgebiet mit einem Freizeit- und Einkaufszentrum von überregionaler Ausstrahlung. Der Ort wurde folglich gerade auch durch die Verbindung zu anderen Orten generiert. Der performative Charakter dieses place-making (Healey 2004) konnte in der Analyse von Originaldokumenten aus dem Planungsprozess nachverfolgt werden. In einer Abstimmung hiess das Berner Stimmvolk 1999 die neue Überbauungsordnung und die mittlerweile auch juridisch gefestigte Public-Private-Partnership gut, ein Grossteil des Projektes ist heute realisiert. Abb. 2 zeigt eine Übersicht zu den einzelnen Teilprojekten: Verschiedenste Akteure und Elemente werden in das Projekt integriert, entfalten in ihrem Zusammenspiel ihre Wirkung und generieren einen neuen Ort, welcher mit der anfänglichen Konzeption von Abb. 1 kaum Gemeinsamkeiten aufweist. Zudem zeigt Abb. 2, wie Brünnen auf Basis mannigfacher Relationen generiert werden soll. Sie illustriert im Vergleich mit Abb. 1 die eingangs argumentierte Komplexitätszunahme in der Planung, die nach adäquaten relationalen Konzeptionen der Analyse verlangt.

\subsection{Cornaredo Lugano}

Entscheidend für das Aufbrechen von Beziehungen und das relationale Generieren eines neuen Ortes - des neues Quartiers Cornaredo - war, dass 1994 im Zusammenhang mit einem neuen regionalen Verkehrsplan beschlossen wurde, Luganos Nordseite mittels eines Strassentunnels mit der Autobahn zu verbinden. Die Verbindung zur Autobahn führt dazu, dass Luganos «Hinterhof» mit den Sportanlagen, dem Friedhof und Abstellflächen für Bauunternehmen quasi zum «Eingangstor» und so die bisherige städtische Struktur auf den Kopf gestellt wird. Während eines Jahrhunderts hatten mentale und materielle Elemente ein relationales Gefüge im Sinne eines Assemblage gebildet und fixiert, welches nun durch einen externen Schock de-territorialisiert wurde und sich im untersuchten Prozess schrittweise und experimentell zu einem neuen Ort zu bilden beginnt. Durch die neue Erschliessung eröffnen sich andere Perspektiven der zukünftigen räumlichen Entwicklung, und zwar sowohl in der Planung im Sinne einer vorwegnehmenden Rahmung der Entwicklung als auch auf Basis der relationalen Materialität des Assemblage Cornaredo. Über das neue Element des Tunnels und die hiermit veränderten Relationen (und der entsprechend generierten Möglichkeitsräume) des Gebietes Cornaredo geraten verschiedene Elemente auf veränderte Weise miteinander in Beziehung - z.B. zentrumsnahe Gemeinden mit bevorzugter Wohnlage, die Städte Lugano und Mailand, der Kanton Tessin sowie potentielle Investoren. Hinsichtlich der Aktualisierung der veränderten Entwicklungspotentiale im Gebiet entsteht eine «intensive Situation» (eine Situation, die vielfältige unterschiedliche potentielle Entwicklungen in sich trägt), welche nicht zuletzt aufgrund der gemeindeübergreifenden Anlage des Gebietes durch einen hohen Grad der Ressourceninterdependenz, eine Vielzahl von Beteiligten und Unsicherheiten bezüglich der Entwicklungsrichtung (Vorgehen und Zielsetzung) gekennzeichnet ist.

Die gewandelten Relationen generieren einen Möglichkeitsraum mit veränderten Tendenzen des Werdens, also mit veränderten Potentialen. Deren Aktualisierung und also eine Re-Territorialisierung der Relationen wurde im Sinne eines Kipp-Punkts ausgelöst, als der Kanton Tessin auf Basis eines Richtplaneintrages des erwähnten Strassenprojektes begann, Entwicklungskonzepte für das Gebiet zu erstellen. Der Kanton Tessin setzte seinen Lösungsweg in der Logik der hierarchischen Verordnung top-down und sektoriell aus der Verkehrsperspektive fort, wie es bei der vorgängigen Verkehrsplanung funktionierte. Mit seiner Initiative schaffte er Unsicherheiten bezüglich Zuständigkeiten und Zielsetzungen, die ihrerseits in einer unantizipierten Dynamik mündeten: Die Gemeinden fühlten sich durch die aktive (Nutzungs-) Planung des Kantons in ihrer Kompetenz beschnitten, was schliesslich zu Rekursen gegen die Pläne des Kantons und damit zum Scheitern des bisherigen Lösungsweges führte. 


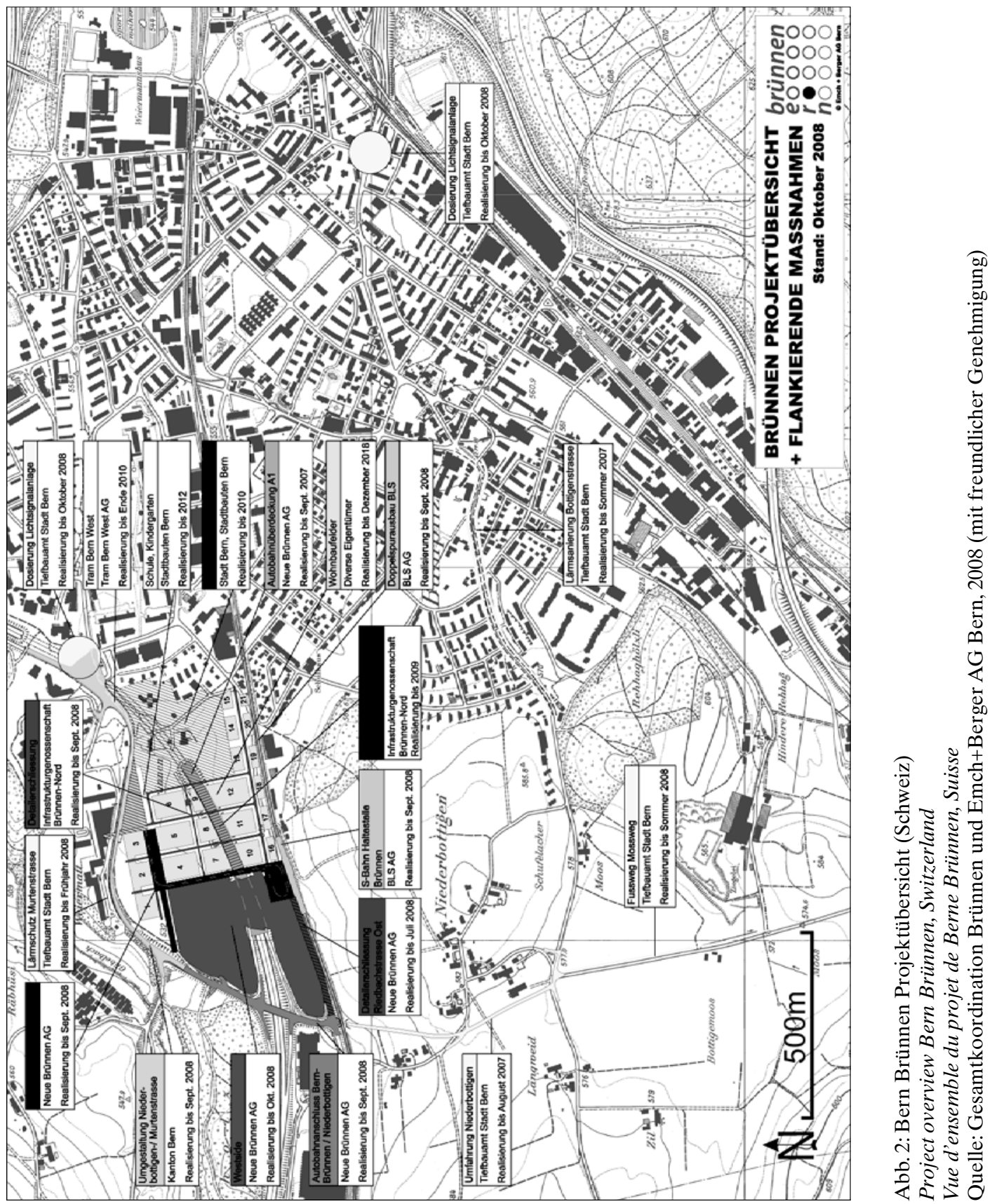




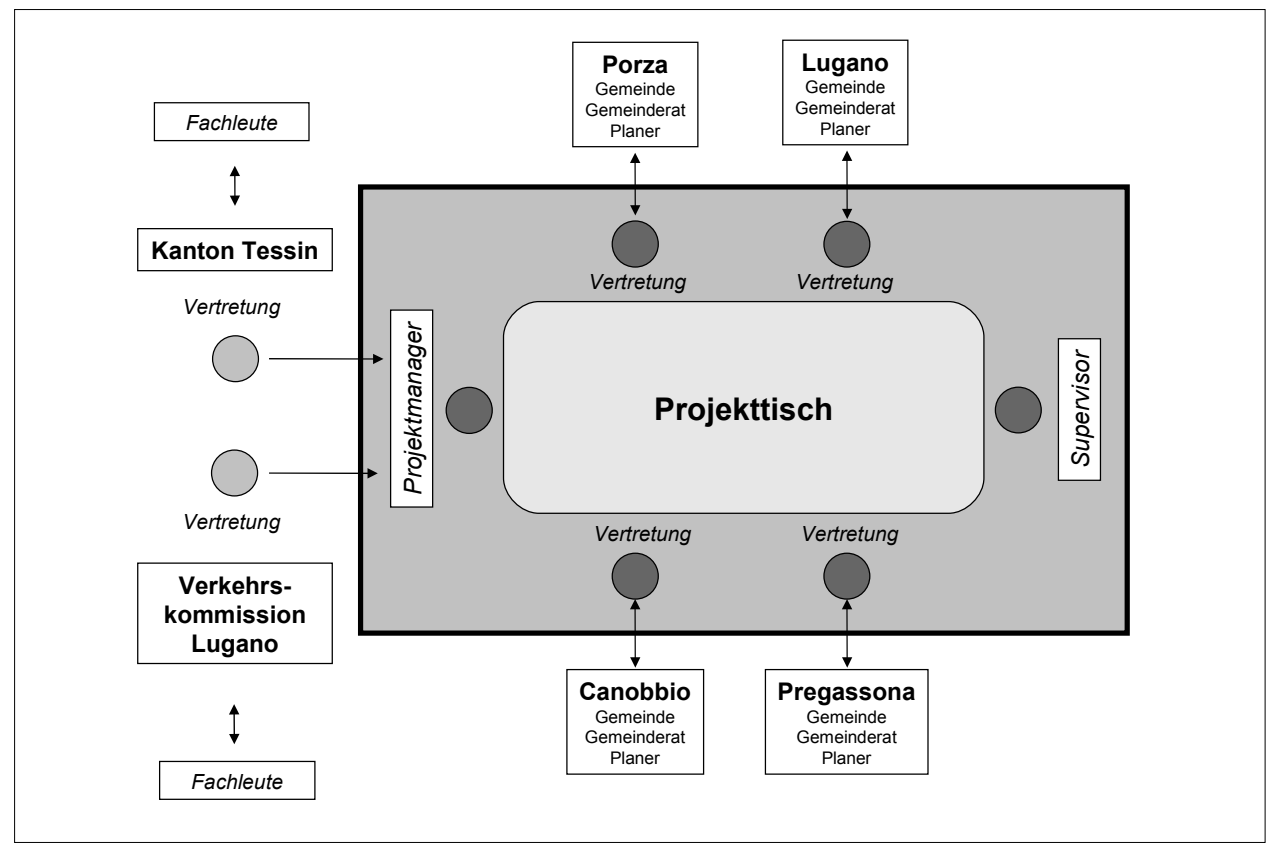

Abb. 3: Projektorganisation Cornaredo Lugano, Schweiz

Project organisation Cornaredo Lugano, Switzerland

Organisation du projet de Cornaredo Lugano, Suisse

Quelle: Studi Associati SA Lugano, 2004 (mit freundlicher Genehmigung)

$\mathrm{Zu}$ diesem Zeitpunkt konnte ein weiterer wichtiger Kipp-Punkt identifiziert werden: Eine Schlüsselfigur - Professor für Wirtschaft an der neuen Universität in Lugano -, die sich an der Schnittstelle von verschiedenen lokalen wirtschaftlichen und politischen Prozessen und damit auch in einer bevorzugten Position dieser Netzwerke und deren Verknüpfung befindet, brachte sich durch Anfrage der Gemeinden ins Spiel und initiierte einen veränderten Lösungsversuch. Ihre Idee bestand darin, die Entwicklung des Gebietes mit der Organisation eines Runden Tisches (tavolo) anzupacken, bei dem die Exekutiven der betroffenen Gemeinden vertreten waren.

Der tavolo liess die verschiedenen Komponenten des Ensembles in eine veränderte Beziehung treten: Die bisher unbeteiligten Gemeinden wurden in der Logik der Verhandlung (siehe oben) in den Prozess der Entscheidungsfindung eingebunden, bislang fragile Relationen wurden gefestigt (territorialisiert). Der Entscheid zur gemeindeübergreifenden Nutzungsplanung stabilisierte den eingeschlagenen Lösungsweg. Der durch die kooperative Herangehensweise weit geöffnete Möglichkeitsraum wurde am Anfang des
Planungsprozesses bewusst offen gehalten und mittels eines Ideenwettbewerbes erkundet. Eine resultierende Stossrichtung wurde schliesslich in einem Masterplan festgehalten (vgl Abb. 3).

Durch die veränderte Beziehung zu Verkehrsinfrastruktur, Entwicklungsplänen, aber auch politischen und planerischen Diskursen wird Cornaredo als ein neuer Ort generiert. Der Projekterfolg hängt wesentlich von der Skalierung des Ortes ab. Die Standortplanung des entstehenden Quartiere di Eventi in Bezug auf die Weltausstellung Milano 2015 zeigt auf, dass die Grösse des Ortes vor allem eine Frage der Relationen und Netzwerke und nicht eine der Nutzungsplanung ist. Globalisierte Zusammenhänge werden durch die relationale Assemblage-Theorie sichtbar gemacht als Konstituenten lokaler Bedingungen und als generative Momente in der Produktion von Orten.

\subsection{Parc Ela}

Der Planungsprozess beim Fallbeispiel Parc Ela wurde 2001 durch eine Informationsveranstaltung des Regionalverbandes Mittelbünden (RVM) angestossen. Dadurch bot sich eine Plattform für den Austausch 


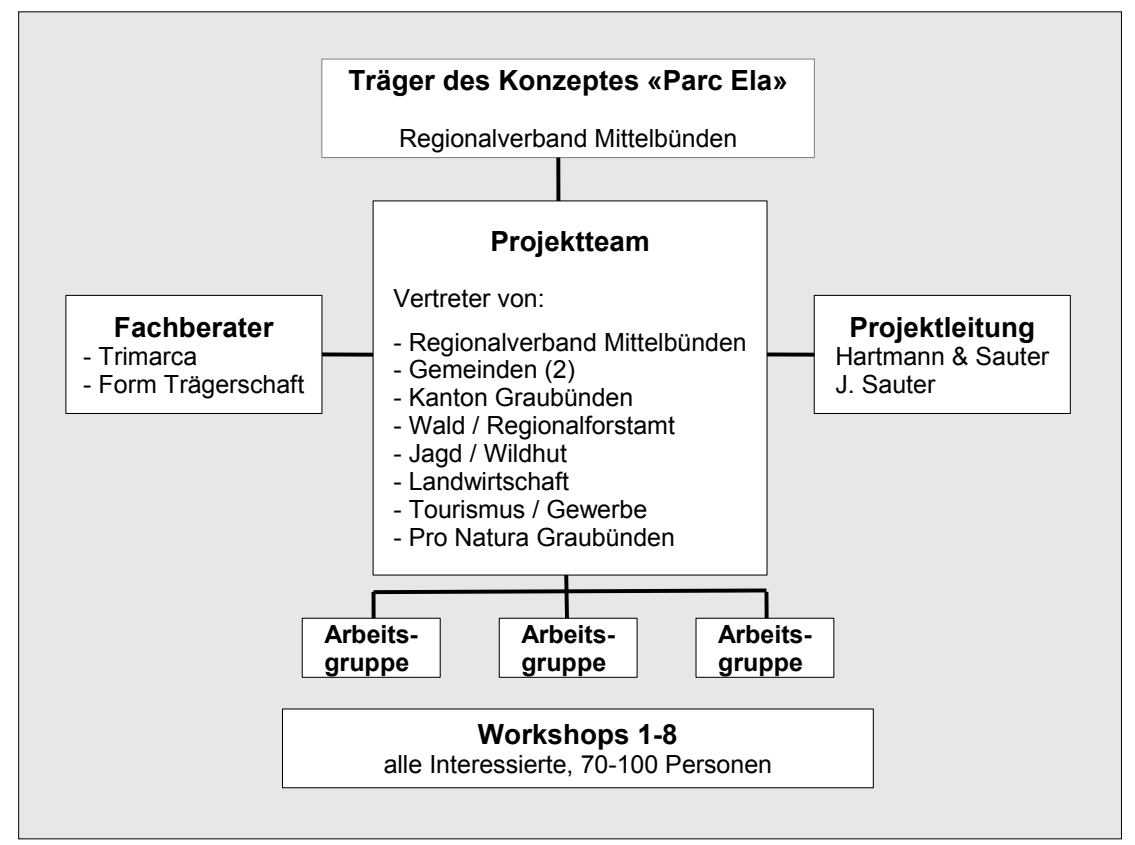

Abb. 4: Projektorganisation Parc Ela, Graubünden, Schweiz (Konzeptphase)

Project organisation Parc Ela, Grisons, Switzerland (conceptual phase)

Organisation du projet du Parc Ela, Grisons, Suisse (phase conceptuelle)

Quelle: eigene Darstellung nach der Originalabbildung von Hartmann \& Sauter, Raum- und Verkehrsplanung Chur, 2002

zwischen verschiedensten Akteuren der Region. Insbesondere ging es in der Anfangsphase um die Kommunikation und das Verständnis der Idee eines Parks, wobei sich der RVM auf das Konzept eines Regionalparks stützte, wie es sich zu dieser Zeit bereits im Luzernerischen Entlebuch in Umsetzung befand. An dieser Stelle gilt es anzumerken, dass sich das Bundesamt für Umwelt zu jener Zeit mit der Revision des Natur- und Heimatschutzgesetzes (NHG) und der dazugehörigen Pärkeverordnung (PäV) beschäftigte. Dabei trugen die Erkenntnisse aus dem Beispiel Parc Ela wesentlich zur Entstehung und Formulierung der Verordnung bei. Auf der anderen Seite stützten sich die Projektverantwortlichen auf die provisorische PäV und erhofften sich dementsprechend auch finanzielle Unterstützung, wodurch der Prozess der Revision für das Projekt direkt relevant wurde. Als vorwiegend politischer Prozess war er mit grosser Unsicherheit behaftet. Der initiierte Prozess der Entscheidungsfindung zeichnete sich im Wesentlichen durch den Aufbau einer breit abgestützten Projektorganisation (vgl. Abb. 4) und ein sehr offenes Prozessdesign aus. So war es auch das Ziel, dass, abgesehen von gewissen Anforderungen an die Qualität, Inhalte des Parks gemeinsam mit der Bevölkerung erarbeitet wurden. Nur so wurde es möglich, die so genannten endogenen Potentiale der Region durch eine Neuordnung von Relationen zu aktualisieren und den Parc Ela auf Basis von Diskursen, rechtlichen Regelungen, lokalen Produktionsnetzen, Bezügen zum Entlebuch und Anderem zu generieren. Resultat der Konzeptphase war eine so genannte Charta, welche die Relationen der internen Organisation unter den Parkgemeinden regelt. Die Charta wurde von den Parkgemeinden einstimmig angenommen, obwohl sie eine finanzielle Regelung mit einer grosszügigen Beteiligung der Gemeinden beinhaltete, um aufgrund der Unsicherheit über die PäV vom Bund unabhängig zu bleiben. Die unsichere Situation verschärfte sich noch weiter, als 2004 die Inkraftsetzung des NGH und der dazugehörigen PäV vom Bundesrat - das Gremium war mittlerweile neu zusammengesetzt, wodurch dessen Koalitionsformen und folglich Entscheide sich wandelten - aufgrund finanzpolitischer Überlegungen aus der Legislaturplanung gestrichen wurde. Dies konnte als Kipp-Punkt im Prozessverlauf identifiziert werden: Das Projekt wurde destabilisiert, nicht zuletzt weil der Wegfall des Elements der Bundesfinanzierung den 
Möglichkeitsraum deutlich veränderte. Dies löste im Parlament, bei den Pärken und bei diversen NichtRegierungs-Organisationen heftige Proteste aus, was den Bundesrat dazu bewegte, 2005 die Botschaft zur Revision doch noch vorzulegen. Ein weiterer KippPunkt im Planungsprozess wird durch den Übergang von der Konzept- zur Umsetzungsphase und damit $\mathrm{zu}$ einer neuen Organisationsform markiert, welche aufgrund einer veränderten personellen Zusammensetzung neue Chancen, aber auch Konflikte mit sich brachte. So konnte beispielsweise ein Botschafter der World Trade Organization (WTO) für das Präsidium der Trägerschaft gewonnen werden, welcher die Verbindung zu einem internationalen Netzwerk verkörpert. Die exterioren Relationen der beteiligten Personen, die gleichzeitig Teil verschiedener Assemblages sind, generierten Potentiale, die in Form der neuen Organisation territorialisiert wurden.

\section{Diskussion: Planerische Lösungsversuche im Umgang mit Unsicherheiten}

Die Analyse der für die Planungsprozesse bestimmenden Momente hat gezeigt, dass für deren Erklärung vielfach Dinge, Relationen und Prozesse miteinbezogen werden müssen, welche auf den ersten Blick nicht als projektrelevant erscheinen. So bewirkten Veränderungen in miteinander in Austausch stehenden Systemen wie z.B. die Verkehrsplanung des Kantons Tessin, die NHG-Revision beim Bund oder die Immobilienkrise eine Veränderung der Möglichkeitsräume der betreffenden Projekte. Diese Veränderung kann aufgrund veränderter Beziehungen zwischen bestehenden Teilen als Prozess der De-/Re-Territorialisierung betrachtet werden.

Dabei waren verschiedenste planerische Lösungsversuche festzustellen, welche dem hohen Grad an Ressourceninterdependenzen, der Vielzahl an Beteiligten und dementsprechenden Unsicherheiten über deren Verhalten in unterschiedlicher Weise begegneten. Lösungsversuche mit den Merkmalen einer top-downPlanung trugen der Komplexität der Problemsituation und damit den Unsicherheiten politischer und wirtschaftlicher Prozesse zu wenig Rechnung und scheiterten.

Die Arbeit mit der Assemblage-Theorie macht insbesondere deutlich, dass Planung ein experimenteller und genuin offener Prozess ist. Denn planerische Lösungen erkunden - über eine Abfolge des Scheiterns und des erneuten Versuchs - experimentell den Möglichkeitsraum, der durch die relationalen Planungssituationen geschaffen wird. Die AssemblageTheorie legitimiert eine pro-aktive Planerrolle und einen kreativen Umgang mit den verfügbaren Res- sourcen. Planung im Sinne vorwegnehmender Rahmung wird lesbar als eine Performanz, welche Relationen temporär zu fixieren und andere aufzubrechen versucht.

Bei den untersuchten Fallbeispielen wurden in Ergänzung zu den bestehenden Verfahren projektspezifische temporäre Organisationsformen - so genannte ad hoc-Organisationen - geschaffen. Sie sind temporäre Fixierungen neu geordneter Relationen von Akteuren in Planungssituationen. Mittels ad hoc-Organisationen wird die Koordination zwischen einer Vielzahl von interdependenten Beteiligten durch eine Konzentration der projektrelevanten Prozesse möglich. Anwendung finden ebenfalls veränderte Prozessdesigns, welche die Lösung am Anfang bewusst offen halten und einer prozessualen Verhandlungslogik unterstellen. Die Trennung zwischen Planung und Umsetzung (Phasenmodell, siehe oben) wird dadurch aufgehoben. Unsicherheiten bezüglich des Verhaltens von Akteuren sowie politischen und wirtschaftlichen Prozessen werden dadurch reduziert, dass diese - etwa in Form von Akteuren, deren Aktionen nicht vorherbestimmt werden können - in der jeweiligen Projektorganisation vertreten sind (z.B. Investoren), oder dass bestimmte Vertreter über ein Wissen über projektrelevante Prozesse verfügen (z.B. Gemeindepräsidenten als Experten lokaler politischer Stimmungen). Kumulationen von Unsicherheiten in Kipp-Punkten machen deutlich, dass diese Punkte gleichzeitig ein generatives Moment als auch ein (vorläufiges) Scheitern des Planungsprozesses in sich tragen. Die bisherige Argumentation legt daher nahe, dass gegenwärtige Überlegungen zur Revision planungsrechtlicher Grundlagen den Prozesscharakter projektorientierter Planungen berücksichtigen sollten.

\section{Entscheidungsprozesse und rechtliche Rahmenbedingungen}

Aufgrund der relationalen Sichtweise und des Fokus auf generative Prozesse geraten Teile und Prozesse ausserhalb des Planungsperimeters als Akteure ins Blickfeld, wodurch plötzliche Umbrüche im Planungsprozess erkenn- und erklärbar werden. Grundsätzlich muss jedes dieser Teile daraufhin geprüft werden, was es im Planungsprozess bewirkt oder bewirken kann. In dieser Perspektive können auch materielle Elemente projektrelevant werden: Der Planungsprozess in Cornaredo Lugano kann beispielsweise nicht ohne das Objekt Strassentunnel erklärt werden; die Autobahnabdeckung in Bern Brünnen «be-wirkt» eine Neuordnung (De-/Re-Territorialisierung) von Relationen und generiert Potentiale im Sinne von Bedingungen des Möglichen, die einen dynamischen Umsetzungsprozess erlauben. 
Auf gleiche Art und Weise kann beispielhaft der Frage nach dem Verhältnis veränderter Entscheidungsprozesse zu den geltenden rechtlichen Rahmenbedingungen nachgegangen werden. Die Frage in der vorgeschlagenen Perspektive der Assemblage-Theorie lautet: Wann und wie manifestieren sich rechtliche Rahmenbedingungen in den jeweiligen Prozessen der Entscheidungsfindung? Welche Möglichkeiten öffnen oder verschliessen sich dadurch? Welche Prozesse der De-/Re-Territorialisierung werden dadurch ausgelöst?

Weil die immer nur temporär fixierten Relationen der Elemente Möglichkeitsräume generieren, kann keine «Wirkungsweise» von Gesetzen als gegeben gelten. Daher muss deren Manifestation in den jeweiligen relationalen Settings empirisch erfasst werden. Wie die aufgeführten Ergebnisse zeigen, zeichnen sich veränderte Prozesse der Entscheidungsfindung durch eine übergreifende Kooperation aus. Daher stellt sich die Frage, welche Rolle rechtliche Rahmenbedingungen im Aushandlungsprozess zwischen verschiedenen interdependenten Beteiligten spielen.

Die Untersuchung hat gezeigt, dass sich verschiedene Elemente des funktionales Raumplanungsrechts im Planungsprozess in einer entscheidenden Weise manifestieren: So hat etwa die LSV die Diskussion über mögliche Entwicklungen im Gebiet Bern Brünnen entscheidend mitbeeinflusst. Sieht man von der Unsicherheit bezüglich der Revision des NHG und der dazugehörigen PäV ab, spielt die PäV beim Parc Ela eine unterstützende Rolle hinsichtlich der aktualisierten Lösung, indem sie die Koordination zwischen den aufgabenrelevanten Akteuren durch gewisse Vorgaben zur Organisation sicherstellt. Durch die PäV wird ein kooperativer Lösungsweg in der Logik der Verhandlung gefördert und unterstützt. Dies ist beim Raumplanungsgesetz (RPG) nicht unbedingt der Fall. Während es mit der Nutzungsplanung für die Umsetzung wichtige Instrumente bereitstellt, spielt es in den wesentlichen Phasen der Entscheidungsfindung keine grosse Rolle und wirkt weder hinderlich noch förderlich.

Das hängt auch damit zusammen, dass veränderte Aushandlungsprozesse, wie sie sich in ad hoc-Organisationen abspielen, im RPG nicht vorkommen. Dementsprechend ist auch deren Prozessablauf nicht definiert. Die Rhythmen der Praxis laufen am Takt des RPG vorbei. So sind die Projekte aufgrund der grundsätzlichen Offenheit des Planungsprozesses und der fehlenden rechtlichen Grundlage bezüglich der Unsicherheiten (Ziele und Mittel nach Christensen 1985) an dieser Stelle am empfindlichsten.

Auf Basis der Analyse und der bisherigen Argumentation ist es möglich, einen potentiellen Interventi- onsraum für eine Gesetzesrevision zu identifizieren und Anforderungen an entsprechende Instrumente $\mathrm{zu}$ formulieren. Impulse könnten ab einem gewissen Schwellenwert durch ein Gefäss (wie z.B. eine legitimierte ad hoc-Organisation) aufgefangen werden. Damit könnte ein Austauschprozess zwischen den Beteiligten initiiert, vorangetrieben und gesteuert werden. Das Instrument würde eine unterstützende sowie eine kontrollierende Rolle spielen. Im Sinne der Aufgabenorientierung erfordern jedoch nicht alle Aufgaben solche Instrumente. Es würde sich um eine Ergänzung im Sinne der Ermöglichung handeln. In Cornaredo und Brünnen hätte etwa die Möglichkeit, Entwicklungsschwerpunkte innerhalb von Agglomerationen zu definieren und ein legitimiertes Gefäss (siehe oben) dafür zu schaffen, in sehr fragilen Planungsphasen unterstützend gewirkt.

Der Ansatz, planerische Lösungsversuche als komplexe Prozesse kollektiver Entscheidungsfindung zu konzipieren und empirisch in ihrer Entfaltung anhand von Veränderungen von Möglichkeitsräumen und Prozessen der De-/Re-Territorialisierung nachzuzeichnen, kann somit auch einen relevanten Beitrag an eine aktuelle politische Diskussion wie der Revision des Raumplanungsgesetzes liefern. Bezugnehmend auf das dem vorliegenden Artikel vorangestellte Zitat von Christensen 1985 wird gefolgert: Planer sollten ihre Vorgehensweisen den jeweiligen Situationen anpassen, und sie können dabei von gesetzlichen Bedingungen behindert oder unterstützt werden.

\section{Literatur}

Albrechts, L. \& S. Mandelbaum (2006): The network society: the new context for planning. - London: Routledge.

Alexander, C. (2002): The nature of order. Band 1. Berkeley, Calif.: Center for Environmental Structure.

Amin, A. (2004): Region unbound: towards a new politics of place. - In: Geografiska Annaler 86, B: 33-44.

Ashby, W.R. (1956). An introduction to cybernetics. - London: Chapman and Hall.

BERTOLINI, L. (2007): Evolutionary urban transportation planning? An exploration. - In: Environment \& Planning A 39, 8: 1998-2019.

Christensen, K.S. (1985): Coping with uncertainty in planning. - In: Journal of the American Planning Association 51, 1: 63-73.

De Roo, G. \& G. Porter (2006): Fuzzy planning - the role of actors in an fuzzy governance environment. - Aldershot: Ashgate.

DeLanda, M. (2006): A new philosophy of society. - London: Continuum.

Deleuze, G. (1994): Difference and repetition. London: Continuum.

Deleuze, G. \& F. Guattari (1987): A thousand pla- 
teaus. Capitalism and schitsophrenia. - Minneapolis: University of Minnesota Press.

Durlauf, S.N. (2005): Complexity and empirical economics. - In: The Economic Journal 115: 225-243.

Healey, P. (2004): Relational complexity and the imaginative power of strategic planning. - In: European Planning Studies 14: 525-546.

Healey, P. (2005): Governance capacity, policy networks, and territorial specifities. - In: Albrechts, L. \& S.J. Mandelbaum (eds): The network society: a new context for planning? - London, New York: Routlede: 307-311.

Hillier, J. (2006): Local planning in times of change: space, integration \& inclusion. - Auckland: New Zealand Planning Institute \& Planning Institute of Australia and New Zealand Congress 2006.

Hillier, J. \& J.E. Van Wezemael (2008a): Tracing the disorderly real: performing civic engagement in a complex world. - In: YANG, K. \& E. BERGRUD (eds): Civic engagement in a network society. - Charlotte, N.C.: Information Age Publishing.

Hillier, J. \& J.E. Van Wezemael (2008b): Empty, swept and garnished: the public finance initiative case of Throckley Middle School. - In: Space and Polity 12, 2: $157-181$

Hillier, J. \& J.E. Van Wezemael (2008c): Opening up what may yet come: performing civic engagement in a complex world. - In: YANG, K. \& E. BERGRUd (eds): Civic engagement in a network society. - Charlotte, N.C.: Information Age Publishing.

IBERT, O. (2003): Innovationsorientierte Planung - Verfahren und Strategien zur Organisation von Innovation. - Opladen: Leske + Budrich.

Ibert, O. (2009): Von «der» Planung zu multiplen Planungen: Strategische Entscheidungen unter Bedingungen hoher Komplexität und geringer Umweltkontrolle. - In: Geographica Helvetica 64, 2: 89-97.

Jessop, B. (1998): The rise of governance and the risks of failure. The case of economic development. - In: International Social Science Journal 50, 155: 29-45.

Jessop, B. (1999): The governance of complexity and the complexity of governance: preliminary remarks on some problems and limits of economic guidance. - www.lancaster.ac.uk/fass/sociology/papers/jessopgovernance-of-complexity.pdf 20.5.2006.

Keller, D.A., Koch, M. \& K. Selle (1996): Planung und Projekte. - In: DISP 126: 37-46.

LATour, B. (2007): Eine neue Soziologie für eine neue Gesellschaft. Einführung in die Akteur-NetzwerkTheorie. - Frankfurt am Main: Suhrkamp.

LAW, J. \& J. URRY (2004): Enacting the social. - In: Economy and Society 33, 3: 390-410.

MaYer, H.-N. (2004): Projekte in der Stadtentwicklung - Chancen und Risiken einer projektorientierten Planung. - In: Jahrbuch StadtRegion (2003): Schwerpunkt: Urbane Regionen. - Opladen: Leske + Budrich: 133-143.
Rommelspacher, T. (1999): Das Politikmodell der IBA Emscher Park. - In: Informationen zur Raumentwicklung 3/4: 157-162.

ScHOLL, B. (1995): Aktionsplanung: Zur Behandlung komplexer Schwerpunktaufgaben in der Raumplanung. - Zürich: vdf Hochschulverlag.

Siebel, W., Ibert, O. \& H.-N. MaYer (1999): Projektorientierte Planung - ein neues Paradigma? - In: Informationen zur Raumentwicklung 3/4: 163-172.

Summers-EfFler, E. (2007): Vortexes of involvement. Social systems as turbulent flow. - In: Philosophy of the Social Sciences 37, 4: 433-448.

Van Wezemael, J.E. (2008): The contribution of assemblage theory and minor politics for democratic network governance. - In: Planning Theory 7, 2: 165185.

Van Wezemael, J.E. (2009a): Housing studies: between romantic and baroque complexity.-In:Housing, Theory \& Society 25, 2 (in print).

VAN WeZemael, J.E. (2009b): Raumentwicklung ohne Autorenschaft? Zur Komplexität kollektiver räumlicher Entscheidungsfindung. - In: Beiträge zur Deutschen Landeskunde (under review).

\section{Zusammenfassung: Veränderte Prozesse der Ent- scheidungsfindung in der Raumentwicklung}

Ausgehend von veränderten Problemstellungen in der aktuellen Raumentwicklung setzt sich der vorliegende Artikel zum Ziel, planerische Lösungsversuche als komplexe Prozesse der Entscheidungsfindung zu konzipieren. Hierbei wird der Umgang mit Unsicherheit als Ausgangspunkt der Überlegungen gewählt. Durch Aufzeigen dreier aktueller, kontextuell deutlich unterschiedlicher Planungsprojekte aus der Schweiz werden deren bestimmende Momente identifiziert und als «Kipp-Punkte» konzipiert. In der Diskussion wird schliesslich die aktuell brisante Frage aufgegriffen, ob und inwiefern die laufende Revision des Raumplanungsgesetzes in der Schweiz auf die Projekt- und Aufgabenorientierung der Planung eingehen kann. Bezugnehmend auf eigene empirische Befunde und auf Karen S. Christensen's Votum, wonach Planer ihre Vorgehensweisen den jeweiligen Situationen anpassen sollten, folgert der Beitrag, dass Planer dabei je nach gesetzlichen Bedingungen behindert oder unterstützt werden.

Schlüsselwörter: Raumentwicklung, Planung, Unsicherheit, Komplexität, Projekt- und Aufgabenorientierung, Schweiz

\section{Summary: Changing processes in decision making in regional planning}

In view of changes currently experienced in regional planning, this article argues for an understanding of regional planning policy making as a complex decision 
making process. Thus, uncertainty becomes the starting point of further deliberation. The article qualitatively describes the development of three recent and very different regional planning projects in Switzerland. It identifies tipping points at key events in each project. Further, it takes up the hotly debated question in Switzerland of the degree to which the current revision of regional planning policy can and should incorporate project and task driven planning. Picking up on the argument forwarded by KAREN S. CHRISTENSEN that planners should be able to adapt their approach to a given situation, the article offers own empirical evidence to show that legal frameworks can support or hinder planners in their attempts to adapt.

Keywords: regional development, planning, uncertainty, complexity, evidence-based policy making, Switzerland

\section{Résumé: Transformations des processus de prise de décision dans le développement territorial}

En s'inspirant des transformations ayant lieu dans le développement territorial actuel, cet article a pour objectif d'analyser les processus de prise de décision complexes aboutissant à des solutions en termes de planification. L'insécurité constitue le point de départ de la réflexion. Trois différents projets de planification entrepris en Suisse sont analysés et permettent de mettre en évidence les phases de leur réalisation et les «points de basculement». L'article traite également de la question brûlante de savoir si et dans quelle mesure la révision en cours de l'aménagement du territoire suisse peut répondre aux enjeux de la planification. En s'appuyant sur des résultats empiriques propres et sur les conclusions de Karen S. Christensen selon laquelle les pratiques des planificateurs devraient s'adapter aux situations contemporaines, l'article conclut en affirmant que les planificateurs sont freinés ou encouragés au fur et à mesure de leur travail par les cadres légaux.

Mots clés: développement territorial, planification, insécurité, complexité du projet et des tâches d'orientation, Suisse

Prof. Dr. Joris E. Van Wezemael, Universität Freiburg, Departement Geowissenschaften, Geographie, Chemin du Musée 4, CH-1700 Freiburg, Schweiz.

e-mail: vanwezemael@unifr.ch

Matthias Loepfe, M.Sc., Geographisches Institut der Universität Zürich, Winterthurerstrasse 190, CH-8057 Zürich, Schweiz.

e-mail: matthias.loepfe@gmail.com

\section{Manuskripteingang/received/manuscrit entré le} 4.1.2009

Annahme zum Druck/accepted for publication/accepté pour l'impression: 18.5.2009 